\title{
Une ethnologue au Jardin des Plantes - dix petits terrains
}

\author{
J. Salomon Cavin \\ Institut de Géographie et Durabilité, Bât Geopolis, Université de Lausanne, 1015 Lausanne, Switzerland \\ Correspondence to: J. Salomon Cavin (joelle.salomoncavin@unil.ch)
}

Published: 3 November 2015

Lizet, B: Une ethnologue au Jardin des Plantes - dix petits terrains, Éditions Petit Génie, Saint-Nazaire, France, 362 pp., EUR 27, ISBN-13: 979-10-93104-05-8, 2015.

Bernadette Lizet est une ethnobotaniste. A la différence de beaucoup de ses prédécesseurs, ou collègues actuels, ses terrains ne sont pas exotiques mais extrêmement familiers, à commencer par son lieu de travail: le Jardin des Plantes à Paris. Ethnologue "du proche et de l'intime", Bernadette Lizet s'intéresse aux plantes, aux animaux et aux hommes qui se côtoient au sein de cette institution.

Dans cet ouvrage, elle propose une rétrospective de son parcours scientifique à travers huit textes publiés entre 1989 et 2014 et deux textes inédits. Bernadette Lizet explique sa démarche en introduction, dévoilant sa passion pour l'ethnobotanique et la chance de la pratiquer au quotidien. Trois axes majeurs traversent son approche "les relations entre la nature et la société, la ville et le vivant, une ethnologie du proche et de l'intime" (p. 8).

A l'instar de ces collègues auxquels elle rend largement hommage, Bernadette Lizet est une pionnière de l'ethnobotanique. Cette adepte des sciences participatives et de l'observation participante a fait évoluer son domaine, carrefour de disciplines, vers l'étude du vivant en ville. "Sauvages dans la ville, de l'inventaire naturaliste à l'écologie urbaine", ouvrage collectif qu'elle a publié avec Wolf et Cecilia en 1999 est une des premières références d'ampleur sur cette question. Elle a participé au bouleversement accompli non seulement dans la recherche sur la nature en ville mais également dans les pratiques jardinières; dans le contexte idéologique de l'écologie et du développement durable, ces dernières font désormais de "la friche paysagée" (Lizet, 2010), de la nature sauvage et des mauvaises herbes, les nouveaux canons esthétiques.
L'ouvrage se compose de trois parties. Sous la bannière "Héritage" (première partie), trois des quatre premiers articles du recueil proposent une histoire de l'ethnobotanique à travers les aventures intellectuelles singulières de ses précurseurs: Auguste Chevalier, Jacques Barrau, Roland Portères et André Georges Haudricourt. Leurs parcours éclairent la longue transition de la "chaire du Muséum des productions végétales d'origine coloniale" créée en 1929 vers le laboratoire d'ethnobotanique en 1963 (p. 105); Bernadette Lizet $\mathrm{y}$ esquisse des figures très contrastées et hautes en couleur de savants qui vont fonder la discipline par "la démonstration (...) que la plante ne peut pas être dissociée de l'analyse de la société dans son écosystème" (p. 139). Paul Jovet, collègue et ami de l'auteure, occupe à l'évidence une place particulière au sein de ces portraits. Pionnier de l'étude de la flore urbaine et théoricien de l'adventice, ce botaniste fut l'un des premiers "à considérer les plates-bandes, les cours et les arrières cours (...) comme autant de territoires dignes d'intérêt" (p. 9). Loin des canons naturalistes qui privilégient encore aujourd'hui la rareté et les milieux épargnés par l'homme, Paul Jovet a apprécié avant les autres les "milieux tripotés" (p. 95), et "la foule des banalités végétales - souvent cosmopolites" (p. 56). La ville, la banlieue parisienne en particulier, a été son terrain de prédilection et la défense de la nature sur le territoire habité, son credo. Alors que les travaux d'inventaires et d'études écologiques sur la faune et la flore qui vivent dans les villes demeurent encore actuellement exceptionnels (Clergeau, 2010), Paul Jovet a réalisé en 1926 le premier inventaire botanique à Paris (Lizet et al., 1999).

Dans le tout premier article du recueil publié dans Ethnographie française en 1989, l'auteure narre depuis le Second Empire la place traditionnellement peu enviable accordée aux herbes folles et autres terrains vagues à Paris. Une telle perspective historique permet de mieux apprécier le tournant 
qui commence alors à se dessiner pour le sauvage en ville, à l'image de la friche apprivoisée du paysagiste Gilles Clément (p. 62). L'histoire de la friche de la Cour des comptes sise dans le Palais d'Orsay est un passage particulièrement savoureux de ce bel article.

Viennent ensuite les "Lieux" (2e partie). Dans "les Herbes folles du Jardin des Plantes", l'auteure dévoile en détail son "terrain" et en démontre le potentiel inépuisable pour l'enquête ethnologique. Il s'agit d'un lieu à la fois extrêmement entretenu et contrôlé mais suffisamment vaste et changeant pour laisser la place à des développements naturels inattendus: "Le vent, la pluie, les oiseaux ou peut-être les enfants et les botanistes qui herborisent, passant outre les barrières, contribuent à disperser chacun à leur manière les graines et les semences" (p. 185). Ainsi, au-delà de la nature très sage des allées à la française du Muséum, Bernadette Lizet scrute, découvre, puis enquête, pour comprendre l'irruption de parcelles non planifiées de nature. Nombre de témoins sont interrogés car 'l'œil du botaniste, du jardinier-botaniste ou du simple curieux attentif au changement, repère ce qui s'installe et prolifère entre deux sarclages dans les parterres; il observe et interprète l'extension des pelouses qui s'ensauvagent par les bordures" (p. 184).

La troisième et dernière partie rassemble des "Histoires" à commencer par l'épopée de Rodolphe et Pélagie, couple d'encombrants hippopotames dont le pedigree génétique incertain empêche le placement dans un autre zoo. Cette histoire rocambolesque entre Paris, Hanovre, Alger et Madrid, donne à comprendre "la montée d'une nouvelle échelle de valeurs (des animaux en captivité) suivant les critères de la génétique des populations" (p. 290). L'ouvrage se conclut par un texte inédit, les balais, chronique des matériaux nécessaires aux travaux quotidiens. On y apprend l'origine du "pique feuilles en tungstène (chinois) et micro vannerie de fil verte" dont la photo est reproduite en couverture de l'ouvrage; rien moins que le "symbole d'un arrangement créatif entre les hommes et une nature profondément perturbée par le grand désordre planétaire" (p. 338). L'importance accordée à cet outil caractérise parfaitement l'approche ethnographique privilégiée par l'auteure: le repérage des objets ordinaires et d'entrées minuscules "qui permettent de penser large" (p. 12).

Le livre se conclut sur une riche bibliographie de vingt pages.

J'ai aimé lire ce livre qui donne envie de scruter les petites choses qui nous entourent. Bernadette Lizet porte un regard bienveillant et curieux sur son environnement. A l'évidence, cette ethnobotaniste aime (nous) raconter des histoires; des histoires de plantes, d'animaux, d'objets, d'hommes et de femmes: chercheurs, soigneurs, jardiniers, vétérinaires, amateurs, visiteurs; des acteurs dont on suit les pérégrinations (voire les exhalations quand ils sont vivants) par exemple dans "la chronique du jardin d'un naturaliste" (2e partie) qui décrit de manière particulièrement savoureuse les joies et les peines de la fine équipe qui reconstruit le jardin des herbes folles de Paul Jovet dans un coin du muséum. La chronique est précise: “(...) le microcosme aquatique faillit bien ne jamais exister sous les deux érables sycomores, où s'échangèrent des propos plutôt vifs. (Mais, sans lui), nous n'aurions pas ressenti les petites joies de l'arrivée des deux poissons rouges en provenance de l'école botanique, bientôt rejoints par des clandestins (des collègues taxidermistes avaient rendu une liberté relative à leurs hôtes d'aquarium). Nous n'aurions pas non plus constaté la persistance du rite très ancien des piécettes offertes par les visiteurs au dieu des fontaines" (p. 235).

L'écriture de Bernadette Lizet mobilise un vocabulaire naturaliste savant qui voisine aisément avec des descriptions imagées de natures: "Alignements impeccablement réglés, frondaisons bien toilettées, massifs savamment composés. La couleur et les rythmes naturels (le vert et le changement saisonnier) le cèdent à l'artifice horticole" (p. 19). Ce voisinage est particulièrement réussi dans le "Maceron et la Mygale"; on y apprend par exemple à apprécier "la petite pimprenelle au goût de concombre (Poterium dictyocarpum Spach.)" sans trop abuser " $d u$ lierre terrestre (Glechoma hederacea L.), car cette petite Labiée des pelouses urbaines dégage sous la dent un arôme d'Eau de Cologne" (p. 311).

Doit-on regretter la longueur de certains textes, amplement émaillés de noms latins, et, parfois l'absence d'introduction ou de conclusion? Non, à l'évidence. Pour le lecteur pressé, souvent peu de raccourcis ou d'explicitations rapides. Si l'on veut parvenir à approcher le monde complexe de la nature en ville, il faut sans aucun doute se laisser guider par le récit déployé de l'ethnobotaniste.

\section{Références}

Clergeau, P.: "Ecologie urbaine et biodiversité", in: Ecologies urbaines, edited by: Coutard, O., Economica, Paris, 154-183, 2010.

Lizet, B.: Du terrain vague à la friche paysagée, Ethnologie française, 4, 40, 597-608, 2010.

Lizet, B., Wolf, A.-E, and Celicia, J. (Eds.): Sauvage dans la ville, de l'inventaire naturaliste à l'écologie urbaine, Publications scientifiques du Muséum d'Histoire naturelle, Paris, 607 pp., 1999. 